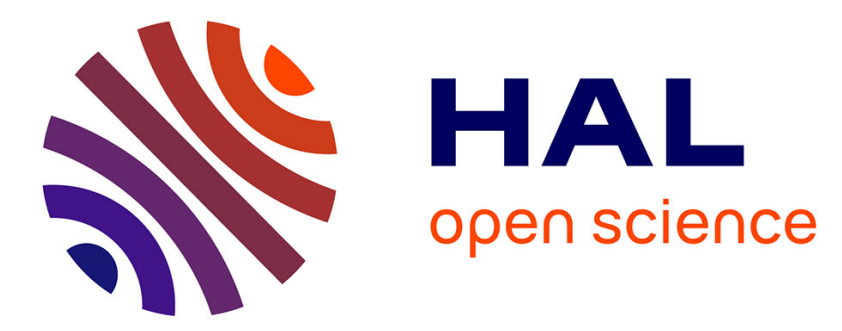

\title{
Modulation spatio-temporelle en spectroscopie des jets moléculaires. Phénomènes au voisinage de la résonance
}

\author{
C. Genty, J.J. Miller, J.G. Theobald
}

\section{To cite this version:}

C. Genty, J.J. Miller, J.G. Theobald. Modulation spatio-temporelle en spectroscopie des jets moléculaires. Phénomènes au voisinage de la résonance. Revue de Physique Appliquée, 1978, 13 (9), pp.457461. 10.1051/rphysap:01978001309045700 . jpa-00244475

\section{HAL Id: jpa-00244475 https://hal.science/jpa-00244475}

Submitted on 1 Jan 1978

HAL is a multi-disciplinary open access archive for the deposit and dissemination of scientific research documents, whether they are published or not. The documents may come from teaching and research institutions in France or abroad, or from public or private research centers.
L'archive ouverte pluridisciplinaire HAL, est destinée au dépôt et à la diffusion de documents scientifiques de niveau recherche, publiés ou non, émanant des établissements d'enseignement et de recherche français ou étrangers, des laboratoires publics ou privés. 


\title{
MODULATION SPATIO-TEMPORELLE EN SPECTROSCOPIE DES JETS MOLÉCULAIRES. PHÉNOMÈNES AU VOISINAGE DE LA RÉSONANCE
}

\author{
C. GENTY, J. J. MILLER et J. G. THEOBALD
}

Laboratoire de Spectroscopie Hertzienne et d'Electronique, Faculté des Sciences, 25030 Besançon, France

(Reçu le 9 mars 1978, révisé le 26 avril 1978, accepté le 8 mai 1978)

\begin{abstract}
Résumé. - On étudie, sur un spectromètre à jet, les phénomènes survenant au voisinage d'une résonance moléculaire, lorsque le champ d'irradiation présente une structure spatiale périodique. La probabilité de transition présente un certain nombre de maxima, variable avec le nombre $n$ de demi-périodes présenté par le champ. Du spectre ainsi obtenu, on peut déduire certaines caractéristiques thermodynamiques du jet moléculaire.
\end{abstract}

\begin{abstract}
The phenomena which occur near the molecular resonance when the irradiation field shows a spatial periodic structure are studied on a beam spectrometer. The transition probability shows a number of maxima variable with the number $n$ of half periods of the field. From the spectrum thus obtained, we can deduce some thermodynamic caracteristics of the molecular beam.
\end{abstract}

1. Introduction. - Dans un article précédent [1] des chercheurs de notre laboratoire ont montré comment, dans un spectromètre à jet moléculaire, le mouvement des molécules à travers une cellule d'irradiation transformait une modulation spatiale $d u$ champ d'irradiation en modulation temporelle. Cette. méthode s'apparente à celle de Hadeishi et al. [2] qui utilisent une modulation résonnante d'un champ continu, créée par le mouvement des molécules. Notre méthode peut être considérée comme une généralisation de celle-ci ; toutefois, on peut remarquer que la technique de Hadeishi conduit à un champ sinusoïdal unique alors que les expériences décrites ici reviennent à soumettre les molécules à une double irradiation par 2 champs sinusoïdaux de pulsation $\omega \pm \Omega$. Ce genre d'expérience présente aussi une analogie avec les expériences de modulation en amplitude ou en fréquence réalisées couramment et depuis longtemps, par exemple en R.P.E. [3] avec cette différence que la fréquence de modulation n'est pas imposée, mais dépend de la vitesse des molécules. La figure 1 montre la configuration de la cellule, constituée de $n$ éléments ( $n$ paires de barres ou $n$ condensateurs plans), le pas du système étant $d$, et chaque élément étant soumis au champ R.F. avec la polarité indiquée. Rappelons que cette cellule fait partie d'un spectromètre à 2 focaliseurs du type Rabi à détection directe par le jet, le détecteur utilisé étant sensible au flux [4]. Une molécule de vitesse $v$ traversant le système voit un champ R.F. de pulsation $\omega$ dont l'amplitude varie avec la pulsation $\Omega=\pi v / d$ et dont

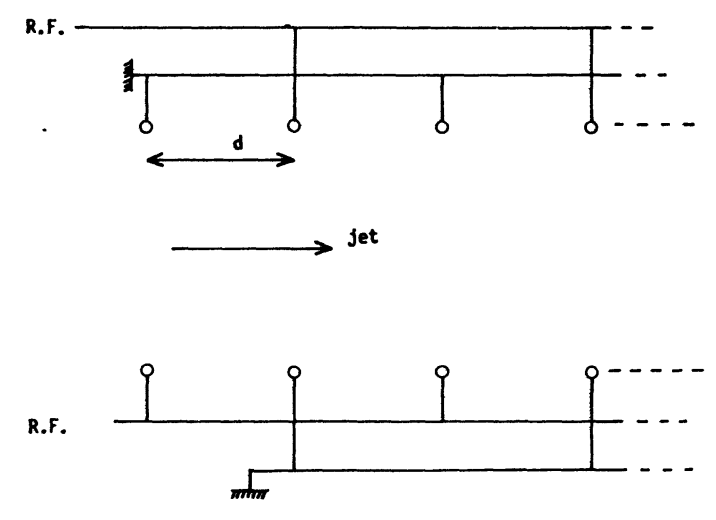

FIG. 1. - Cellule de modulation.

[Modulation cell.]

l'expression, sur l'axe du système est, avec une très bonne approximation [1] $e=\varepsilon \sin \omega t$, avec $\mathcal{E}=E \sin \Omega t$ où $E$ est l'amplitude du 1 er terme de Fourier du champ créé par la cellule.

L'étude citée en référence [1] avait été faite au moyen d'une cellule comportant un nombre d'éléments relativement grand $(n=27)$, présentant quelques inhomogénéités de champ. Par ailleurs, le jet était tel qu'une fraction non négligeable des molécules traversait la cellule assez loin de l'axe, dans des zones où le champ présente une composante d'amplitude constante.

Les travaux qui font l'objet de cet article affinent les résultats expérimentaux pour $n$ grand, par une construction mécanique plus soignée de la cellule et 
par un jet diaphragmé mieux localisé au voisinage de l'axe. Mais surtout, nous mettons en évidence, lorsque $n$ est petit, une évolution nouvelle de la probabilité de transition.

2. Probabilité de transition. - Nous utilisons la transition $4_{3}$ de dédoublement $\mathrm{K}$ du formol (fréquence moléculaire égale à $4,573 \mathrm{MHz}$ ) du type dipolaire électrique. Chaque molécule constitue un système à 2 niveaux d'énergie $E_{1}=\hbar \omega_{1}$ et $E_{2}=\hbar \omega_{2}$, valeurs propres de l'hamiltonien non perturbé $H_{0}$, d'états propres $\left|\varphi_{1}\right\rangle$ et $\left|\varphi_{2}\right\rangle$. Dans la cellule d'irradiation, la molécule est soumise au champ R.F., soit à la perturbation $W(t)$ et l'état du système à l'instant $t$ est décrit, dans l'approximation des états stationnaires, par

$$
|\psi\rangle=b_{1}(t) \mathrm{e}^{-i \omega_{1} t}\left|\varphi_{1}\right\rangle+b_{2}(t) \mathrm{e}^{-i \omega_{2} t}\left|\varphi_{2}\right\rangle .
$$

Le $1^{\mathrm{er}}$ focaliseur focalisant les états supérieurs et éliminant les états inférieurs des doublets, à l'instant $t=0$ où les molécules pénètrent dans la cellule d'irradiation, on a, $\left|b_{1}(0)\right|^{2}=1$ et $\left|b_{2}(0)\right|^{2}=0$ et la probabilité de transition au temps $t$ de $\left|\varphi_{1}\right\rangle$ vers $\left|\varphi_{2}\right\rangle$ est donnée par $P(t)=\left|\left\langle\varphi_{2} \mid \psi\right\rangle\right|^{2}=\left|b_{2}(t)\right|^{2}$.

En supposant que $W(t)$ reste petite, la théorie des perturbations au $1^{\mathrm{er}}$ ordre conduit à (rappelons qu'il n'y a pas de relaxation)

$$
i \hbar \frac{\mathrm{d} b_{2}}{\mathrm{~d} t}=W \mathrm{e}^{i \omega_{21} t} \quad \text { où } \quad W=\left\langle\varphi_{1}|W(t)| \varphi_{2}\right\rangle .
$$

Si $\mu$ est le moment dipolaire électrique de la molécule,

$$
W=-\left\langle\varphi_{1}|\mu E| \varphi_{2}\right\rangle \sin \Omega t \sin \omega t
$$

et l'équation précédente s'écrit :

$$
\frac{\mathrm{d} b_{2}}{\mathrm{~d} t}=\frac{\beta}{i}\left[\mathrm{e}^{i(\Delta+\Omega) t}-\mathrm{e}^{i(\Delta-\Omega) t}\right]
$$

avec

$$
\beta=\frac{\left\langle\varphi_{1}|\mu E| \varphi_{2}\right\rangle}{4 \hbar} \text { et } \Delta=\omega-\omega_{12}
$$

d'où

$$
b_{2}\left(t_{n}\right)=\frac{1-\mathrm{e}^{i(\Delta+\Omega) t_{n}}}{\Delta+\Omega}-\frac{1-\mathrm{e}^{i(\Delta-\Omega) t_{n}}}{\Delta-\Omega}
$$

$t_{n}$ étant le temps mis par une molécule de vitesse $v$ pour traverser le système de $n$ éléments.

La probabilité de transition pour ces molécules de vitesse $v$ est alors :

$$
P_{v}(\Delta)=4 \beta^{2}\left\{\frac{\sin ^{2}\left(\frac{n \pi}{2} \frac{\Delta}{\Omega}+\frac{n \pi}{2}\right)}{(\Delta+\Omega)^{2}}+\frac{\sin ^{2}\left(\frac{n \pi}{2} \frac{\Delta}{\Omega}-\frac{n \pi}{2}\right)}{(\Delta-\Omega)^{2}}-\frac{2 \sin ^{2} \frac{n \pi}{2} \frac{\Delta}{\Omega}-\sin ^{2} \frac{n \pi}{2}}{\Delta^{2}-\Omega^{2}} \cos n \pi\right\}
$$

expression qui se réduit à

$$
\begin{aligned}
& P_{v}(x)=\frac{16 \beta^{2} d^{2} v^{2}}{\pi^{2}} \cdot \frac{\sin ^{2} \frac{n \pi \mathrm{d} x}{v}}{\left(4 d^{2} x^{2}-v^{2}\right)^{2}} \quad \text { pour } n \text { pair }, \\
& P_{v}(x)=\frac{16 \beta^{2} d^{2} v^{2}}{\pi^{2}} \cdot \frac{\cos ^{2} \frac{n \pi \mathrm{d} x}{v}}{\left(4 d^{2} x^{2}-v^{2}\right)^{2}} \quad \text { pour } n \text { impair },
\end{aligned}
$$

$\operatorname{avec} x=\Delta / 2 \pi$.

Ces expressions, valables pour un jet monocinétique, montrent que la probabilité de transition varie de façon sinusoïdale, le spectre présentant 2 raies principales pour $x \simeq \pm v / 2 d$ quel que soit $n$, et des raies secondaires pour

$$
\begin{array}{ll}
x \simeq \frac{2 k+1}{n} \cdot \frac{v}{2 d} & \text { si } n \text { est pair }, \\
x \simeq \frac{2 k}{n} \cdot \frac{v}{2 d} & \text { si } n \text { est impair } .
\end{array}
$$

Elle est nulle pour

$$
x=\frac{2 k}{n} \cdot \frac{v}{2 d}, \quad k \neq \pm \frac{n}{2} \text { si } n \text { pair }
$$

et

$$
x=\frac{2 k+1}{n} \cdot \frac{v}{2 d}, \quad k \neq \frac{n-1}{2} \text { si } n \text { est impair . }
$$

En réalité, les 2 raies secondaires situées de part et d'autre des raies principales sont masquées par ces dernières, et dans le cas de $n=1$ les 2 raies principales et la raie secondaire centrale ne sont pas résolues et forment une raie unique centrée à $x=0$. Ces résultats sont illustrés par les courbes théoriques de la figure 2 . (Les spectres étant symétriques, une seule moitié est représentée.)

Dans la pratique, le jet n'est pas monocinétique, 


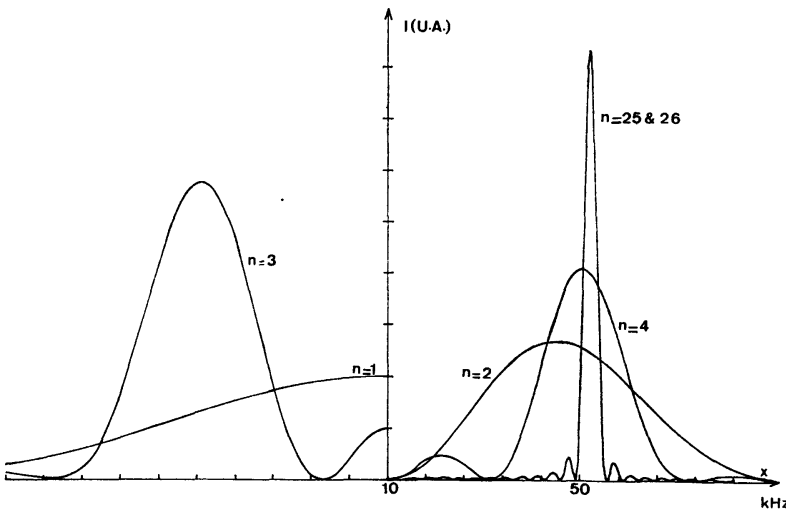

FIG. 2. - Spectres monocinétiques $v=630 \mathrm{~m} / \mathrm{s}$ pour $n=1,2,3$, 4,25 et 26.

[Monokinetic spectra $v=630 \mathrm{~m} / \mathrm{s}$ for $n=1,2,3,4,25$ and 26.]

mais présente une distribution longitudinale des vitesses $J(v)$, et la probabilité de transition $P_{v}$ doit être remplacée par une moyenne sur cette distribution, soit

$$
\langle P\rangle=\int_{0}^{\infty} J(v) P(v) \mathrm{d} v .
$$

Pour ce qui nous concerne, notre source n'est pas strictement effusive, le jet étant formé dans ces expériences à partir d'un canal cylindrique de $7 \mathrm{~mm}$ de longueur et $1 \mathrm{~mm}$ de diamètre, la pression source étant $p_{\mathrm{s}}=266,6 P_{\mathrm{a}}(2 \mathrm{~T})$ et la pression dans l'enceinte $P=0,1333 \times 10^{-3} P_{\mathrm{a}}\left(10^{-6} \mathrm{~T}\right)$. Dans ces conditions, la formation du jet s'accompagne d'une détente lui conférant une vitesse d'ensemble $U$ (cette hypothèse sera vérifiée expérimentalement ci-dessous). La fonction de distribution à employer est alors [5-6]

$$
J(v)=\frac{2 v^{3}}{\alpha^{4}} \mathrm{e}^{-((v-U) / \alpha)^{2}}
$$

$\alpha$ étant la vitesse la plus probable dans la source, et la probabilité de transition s'écrit :

$$
\begin{array}{r}
\langle P(x)\rangle=\frac{32 \beta^{2} d^{2}}{\pi^{2} \alpha^{4}} \int_{0}^{\infty} v^{5} \mathrm{e}^{-((v-U) / \alpha)^{2}} \times \\
\quad \times \frac{\left(\begin{array}{c}
\sin \\
\cos
\end{array}\right)^{2}\left(\frac{n \pi \mathrm{d} x}{v}\right)}{\left(4 d^{2} x^{2}-v^{2}\right)^{2}} \mathrm{~d} v
\end{array}
$$

(sin pour $n$ pair, cos pour $n$ impair).

Les courbes de la figure 3 donnent l'évolution de $\langle P\rangle$ en fonction de $x$ pour diverses valeurs de $n$, avec $U=630$ et $\alpha=155 \mathrm{~m} / \mathrm{s}$.

Pour $n$ petit, seules subsistent les raies principales et les raies secondaires situées à l'intérieur de l'intervalle défini par ces dernières, si bien que le spectre présente un nombre de raies égal au nombre d'éléments de la cellule d'irradiation.

Lorsque $n$ augmente, l'amplitude des raies secondaires diminue pour s'annuler lorsque $n$ est grand et

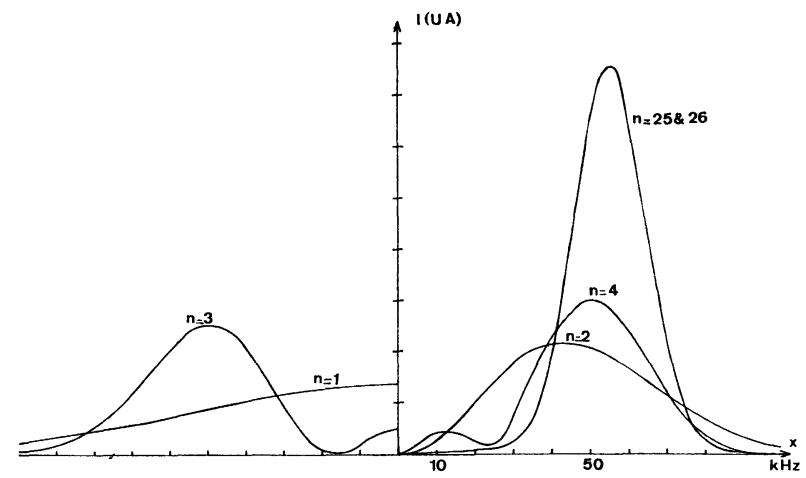

FIG. 3. - Spectres en $J(v)$ pour $n=1,2,3,4,25$ et 26 .

[ $J(v)$ spectra for $n=1,2,3,4,25$ and 26.]

le spectre ne comporte plus alors que les 2 raies principales.

Quant à la position de ces raies, elle n'est plus donnée, comme dans le cas d'un jet monocinétique, par une relation analytique simple, même de façon approchée. Le spectre est toujours symétrique par rapport à $x=0$, mais la position des raies dépend à la fois de $U$ et de $\alpha$. Il en est de même pour leur largeur qui est aussi une fonction du couple $(U, \alpha)$.

Cette dépendance a été étudiée pour $n$ grand (égal à 26), (le spectre étant alors le plus simple possible car les raies secondaires ont disparu), sur les raies principales. Les courbes de la figure 4 donnent la position $x_{\mathrm{M}}$ d'une raie principale en fonction de $U$ et de $\alpha$. On y voit qu'elle dépend beaucoup plus de $U$ que de $\alpha$. Les conclusions sont inversées pour la largeur
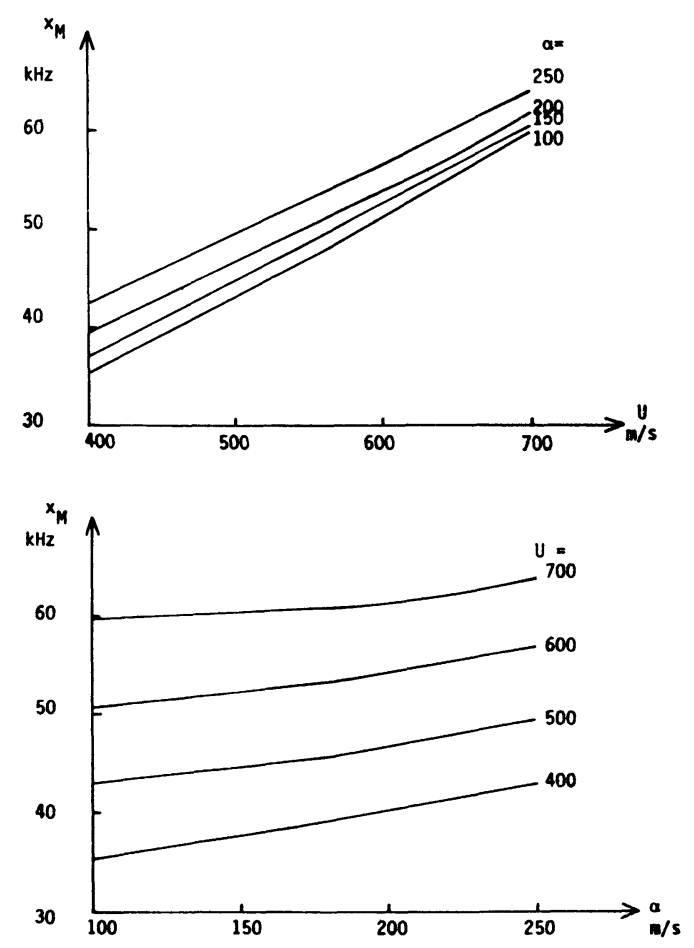

Fig. 4. - Variation de $x_{\mathrm{M}}$ pour $n=26$ en fonction de $U$ (paramètre $\alpha$ ) et de $\alpha$ (paramètre $U$ ).

[Variation of $x_{\mathrm{M}}$ for $n=26$ as a function of $U$ ( $\alpha$ parameter) and of $\alpha$ ( $U$ parameter).] 
à mi-hauteur $\delta$, comme le montrent les courbes de la figure 5 qui donnent $\delta$ en fonction de $U$ et de $\alpha$.
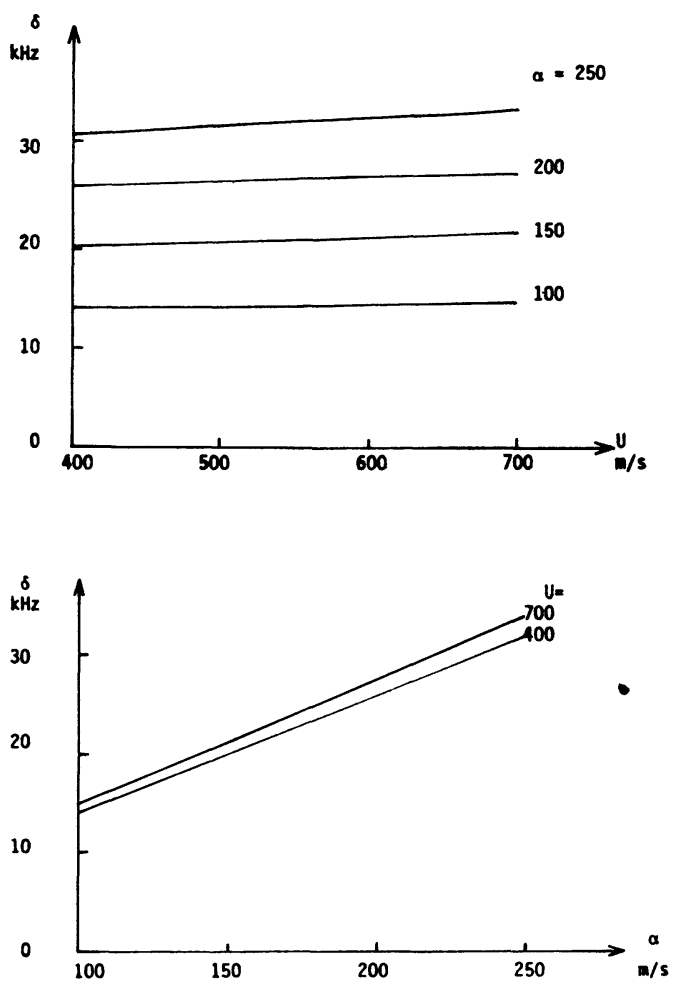

Fig. 5. - Variation de $\delta$ pour $n=26$ en fonction de $U$ (paramètre $\alpha$ ) et de $\alpha$ (paramètre $U$ ).

[Variation of $\delta$ for $n=26$ as a function of $U$ ( $\alpha$ parameter) and of $\alpha$ ( $U$ parameter $)$.]

L'abaque de la figure 6 qui donne $x_{\mathrm{M}}$ en fonction de $\delta$ résume ces résultats et montre qu'il n'existe qu'un seul couple $(U, \alpha)$ permettant d'obtenir une position et une largeur de raie données.

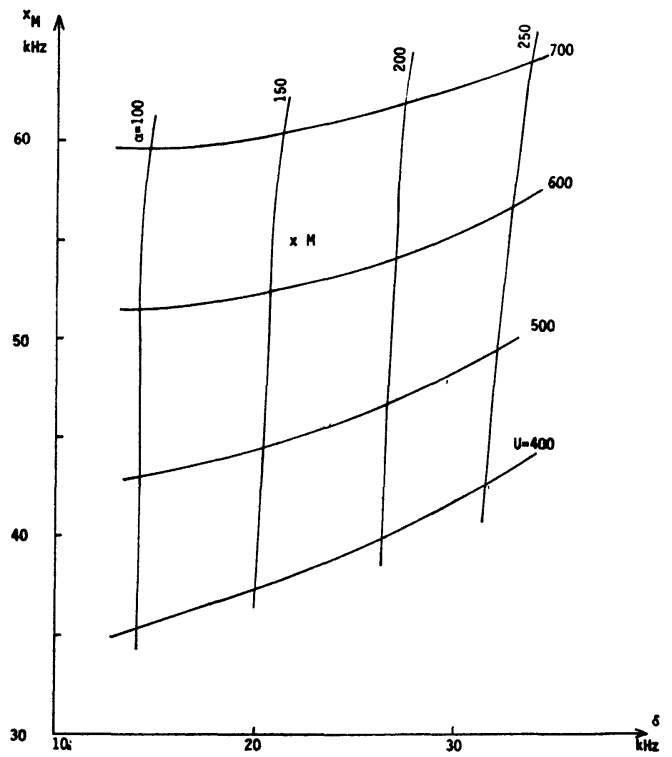

Fig. 6. - Abaque représentant les valeurs $\alpha$ et $U$ en fonction de $x_{\mathrm{M}}$ et $\delta$ pour $n=26$.

[Abaque showing the values of $\alpha$ and $U$ as a function of $x_{\mathrm{M}}$ and $\delta$ for $n=26$.]
Remarque. - $\mathrm{Si}$ on suppose que le jet est formé sans détente $(U=0)$ le spectre ne présente plus que les raies principales et la raie centrale pour $n$ impair, les autres maximums secondaires n'étant pas résolus (Fig. 7).

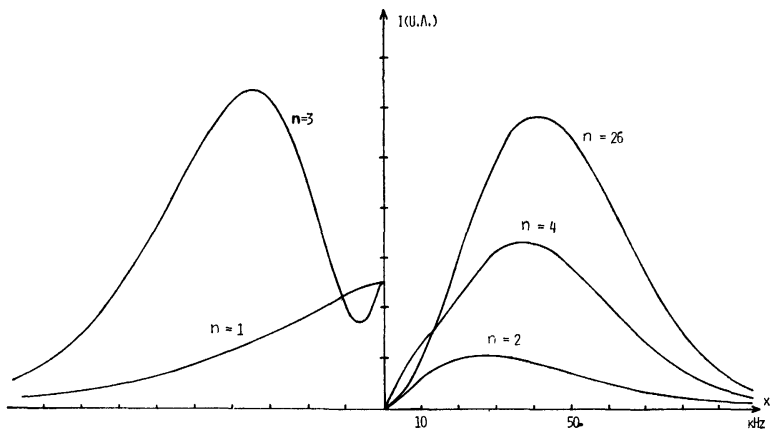

Fig. 7. - Spectre en $J(v)$ avec $U=0$ pour $n=1,2,3,4,25$ et 26 .

[ $J(v)$ spectrum with $U=0$ for $n=1,2,3,4,25$ and 26.]

3. Résultats expérimentaux. - Les cellules d'irradiation utilisées comportent $1,2,3$ et 4 éléments avec $d=3 \mathrm{~cm}$ et 26 éléments avec $d=0,6 \mathrm{~cm}$. Les enregistrements sont représentés sur la figure 8.

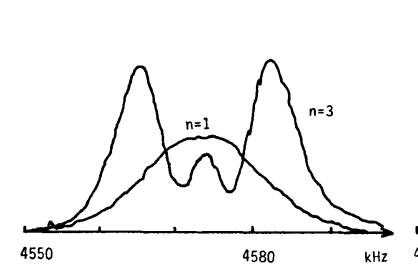

(a)

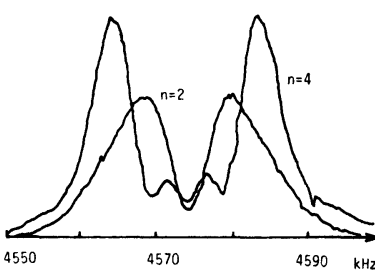

(b)

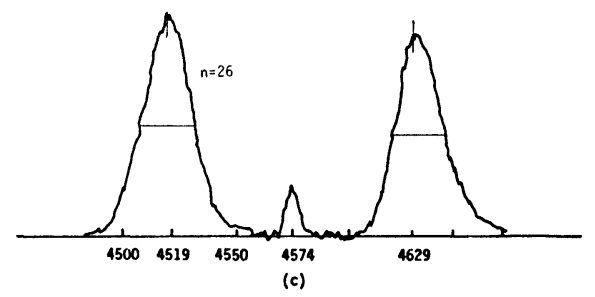

FIG. 8. - Enregistrements des spectres expérimentaux : a) $n=1$ et $3 ; b) n=2$ et $4 ; c) n=26$.

[Recordings of experimental spectra :a) $n=1$ and $3 ; b) n=2$ and $4 ; c) n=26$.]

Sur l'enregistrement $n=26$, les caractéristiques des raies principales sont les suivantes :

$$
\begin{aligned}
x_{\mathrm{M}} & =33 \pm 1 \mathrm{kHz} \\
\delta & =21,5 \pm 1 \mathrm{kHz} .
\end{aligned}
$$

L'utilisation de l'abaque conduit au couple :

$$
U=630 \mathrm{~m} / \mathrm{s}, \quad \alpha=155 \mathrm{~m} / \mathrm{s}
$$

représenté par le point $\mathrm{M}$ sur la figure 6 .

On trace alors le spectre théorique pour $n=1,2,3$ et 4 avec ce couple $(U, \alpha)$. La comparaison entre valeurs théoriques et expérimentales de $x_{\mathrm{M}}$ et $\delta$ pour 
les raies principales est faite sur le tableau I. La concordance de l'ordre de $0,5 \mathrm{kHz}$ entre valeurs théoriques et expérimentales est en accord avec la précision des pointés. Pour $n=4$, les raies secondaires sont situées à $3,2 \pm 0,5 \mathrm{kHz}$ du centre du spectre, alors que la valeur théorique est de $2,7 \mathrm{kHz}$.

\section{TABLEAU I}

Valeurs théoriques et expérimentales de $x_{\mathrm{M}}$ et $\delta$

[Theoretical and experimental values of $x_{M}$ and $\delta$.]

\begin{tabular}{|c|c|c|c|c|c|}
\hline & \multicolumn{2}{|c|}{$d=0,03 \mathrm{~m}$} & & & $d=0,006 \mathrm{~m}$ \\
\hline$x_{\mathrm{M} \text { théor. }}(\mathrm{kHz})$ & $n=1$ & $\begin{array}{c}n=2 \\
6,9\end{array}$ & $\begin{array}{c}n=3 \\
9,3\end{array}$ & $\begin{array}{c}n=4 \\
10\end{array}$ & $\begin{array}{c}n=26 \\
55\end{array}$ \\
\hline M exp. & & $6,2 \pm 1$ & $8,6 \pm 1$ & $9,5 \pm 1$ & $55 \pm 1$ \\
\hline $\begin{array}{l}\delta_{\text {théor. }} \\
\delta_{\text {exp. }}\end{array}$ & $\begin{array}{l}13 \\
17\end{array}$ & $\begin{array}{c}10,2 \\
9,8 \pm 1\end{array}$ & $\begin{array}{c}7,1 \\
7,3 \pm 1\end{array}$ & $\begin{array}{c}6,6 \\
6,5 \pm 1\end{array}$ & $\begin{array}{c}21,5 \\
21,5 \pm 1\end{array}$ \\
\hline
\end{tabular}

4. Conclusion. - Par rapport au travail présenté en référence [1], l'amélioration expérimentale apportée ici a permis de diminuer considérablement, pour $n$ grand, la composante centrale due à des transitions à la fréquence moléculaire sous l'effet du champ résiduel.
L'étude en fonction de $n$ nous a permis d'observer l'évolution de la probabilité de transition quand le nombre d'éléments augmente, et de retrouver les résultats antérieurs comme cas limite. L'utilisation d'une telle cellule à structure périodique semble par ailleurs un moyen commode d'approfondir notre connaissance du jet par la détermination de $U$ et de $\alpha$.

La méthode utilisée étant exclusivement spectroscopique et n'utilisant pas de disques tournants, les inconvénients liés aux méthodes mécaniques sont éliminés; l'analyse des résultats est par ailleurs plus simple [7]. Dans le cas des jets supersoniques obtenus, par exemple, par échange de charges, elle permettrait une mesure directe de l'énergie des particules [8].

Toute méthode d'analyse étant une méthode de sélection le dispositif décrit permet de réaliser une sélection des vitesses, la résolution augmentant avec $n$ [9].

Enfin nous avons traité ici le problème dans l'approximation des champs faibles. Lorsque le champ augmente et que $\beta$ devient de l'ordre de $\Omega$, de nouveaux phénomènes apparaissent : déplacements radiatifs, saturation, transitions à plusieurs photons; ils feront l'objet de publications ultérieures.

\section{Bibliographie}

[1] Annabi, M., Gillet, D., Revue Phys. Appl. 8 (1973) 1.

[2] Hadeishi, T., Bichel, W. S., Garcia, J. D. and Berry, H. G., Phys. Rev. Lett. 23 (1969) 65.

[3] La résonance magnétique nucléaire, GRIVET, P., C.N.R.S. 1955; Autler, S. M. and Townes, C. H., Phys. Rev. 100 (1955) 703.

[4] Genty, C., Theobald, J. G., Revue Phys. Appl. 8 (1973) 361.

[5] Berthier, J. P., Constans, A., Daury, G. et Lostis, P., Revue Phys. Appl. 6 (1971) 433.
[6] DyKe, T. R., TOMASEvich, G. R., KLEMPereR, W., FALCONER, W. E., J. Chem. Phys. 57 (1972) 2277.

[7] Marcus, P. M. and McFee, J. M. in EstermanN, J., Recent Research in molecular beams (Ac. Press. New York) 1959.

[8] Delmas, M., Gautherin, G., Lejeune, C., 4e symposium International sur les jets moléculaires, Cannes 1973, 83.

[9] Annabi, M., Genty, C., Miller, J. J., Theobald, J. G., C.R. Hebd. Séan. Acad. Sci. 285 (1977). 